\title{
Capacidade funcional e perda sensorial em um grupo de idosos usuários de um plano
}

\section{de saúde}

\author{
Functional capacity and sensory loss in a group of elderly users of a health plan \\ Capacidad funcional y pérdida sensorial en un grupo de usuarios mayores de un plan de salud
}

Recebido: 23/01/2021 | Revisado: 31/01/2021 |Aceito: 01/02/2021 | Publicado: 09/02/2021

\author{
Ana Carla Oliveira Garcia \\ ORCID: https://orcid.org/0000-0003-4664-0073 \\ Pontifícia Universidade Católica de São Paulo, Brasil \\ E-mail: anacarlagarciausa@gmail.com \\ Teresa M. Momensohn dos Santos \\ ORCID: https://orcid.org/0000-0003-4751-0721 \\ Pontifícia Universidade Católica de São Paulo, Brasil \\ E-mail): tmomensohn@gmail.com \\ Maria Elisa Gonzalez Manso \\ ORCID: https://orcid.org/0000-0001-5446-233X \\ Pontifícia Universidade Católica de São Paulo, Brasil \\ E-mail: mansomeg@hotmail.com
}

\begin{abstract}
Resumo
Trata-se de pesquisa, exploratória, transversal, realizada com 314 pessoas idosas não institucionalizadas e vinculadas a um programa de gerenciamento de doenças crônicas patrocinado por uma operadora de planos de saúde localizada na cidade de São Paulo, cidade de moradia destas pessoas, durante o período compreendido entre o segundo semestre de 2018 e primeiro de 2019. O objetivo, após comparar grupos de idosos sem e com perdas sensoriais -visual, auditiva e dupla perda-, foi verificar a interação destas com variáveis relacionadas à capacidade funcional e detectar quais fatores influenciam a presença de dupla perda. Coletados dados demográficos, sobre quais e quantas doenças crônicas, número de medicamentos de uso contínuo, presença de perda visual e/ou auditiva diagnosticada e em acompanhamento por médico ou fonoaudiólogo, foram aplicadas escalas para avaliação da capacidade funcional destas pessoas. Após análise descritiva, o grupo foi segmentado a fim de atingir os objetivos da pesquisa, utilizando-se os testes Qui-Quadrado, Exato de Fisher e t-Student. Após realizou-se regressão logística que possibilitou examinar as variáveis com maior influência em relação às perdas sensoriais isoladas e à DPS (Odds ratio). As perdas sensoriais mostraram-se importante que compromete a capacidade funcional, afetando o desempenho para atividades básicas e restringindo a mobilidade. A viuvez associada à dupla perda foi um achado inédito. Para este grupo, o avançar da idade foi um fator preditor importante para o surgimento de perdas sensoriais, principalmente a Dupla Perda Sensorial e ter uma perda prévia, auditiva ou visual, potencializa o risco para seu desenvolvimento.
\end{abstract}

Palavra-chave: Assistência a idosos; Perda auditiva; Cegueira; Dupla perda sensorial; Pessoas com deficiência.

\begin{abstract}
This is an exploratory, cross-sectional study carried out with 314 non-institutionalized elderly people linked to a chronic disease management program sponsored by a health plan operator located in the city of São Paulo, where these people live, during between the second semester of 2018 and the first of 2019. The objective, after comparing groups of elderly people with and without sensory losses - visual, auditory and double loss -, is to verify their interaction with variables related to functional capacity and detect which factors influence the presence of double loss. Data was collected related to which and how many chronic diseases were present, the number of medications of continuous use normally taken, the presence of visual and / or hearing loss diagnosed and constant monitoring by a doctor or speech therapist. Were applied scales to assess the functional capacity of these people. After descriptive analysis, the group was segmented in order to achieve the research objectives, using the Chi-Square, Fisher's Exact and t-Student tests. Afterwards, logistic regression was performed, which made it possible to examine the variables with the greatest influence in relation to isolated sensory losses and DPS (Odds ratio). Sensory losses proved to be significant in compromising functional capacity, affecting performance for basic activities and restricting mobility. Widowhood associated with the double loss was an unprecedented finding. For this group, advanced age was an important predictive factor for the appearance of sensory losses, especially Double Sensory Loss and having a previous hearing or visual loss increases the risk for their development.
\end{abstract}

Keywords: Old age assistance; Hearing loss; Blindness; Double sensory loss; Disabled persons.

\section{Resumen}

Este es un estudio exploratorio, transversal, realizado con 14 ancianos no institucionalizados vinculados a un programa de manejo de enfermedades crónicas auspiciado por un operador de plan de salud ubicado en la ciudad de São Paulo, donde viven estas personas, durante el período de tiempo comprendido entre el segundo semestre de 2018 y el primero de 2019 . El objetivo, 
luego de comparar grupos de personas mayores con y sin pérdidas sensoriales - visual, auditiva y doble pérdida -, fue verificar su interacción con variables relacionadas con la capacidad funcional y detectar qué factores influyen en la presencia de doble pérdida. Se recogieron datos sobre qué y cuántas enfermedades crónicas, número de medicamentos de uso continuo, presencia de perdida visual y / o auditiva diagnosticada y en seguimiento por un médico o logopeda y se aplicaron escalas para evaluar la capacidad funcional de estas personas. Luego después del análisis descriptivo, el grupo fue segmentado para lograr los objetivos de la investigación, utilizando las pruebas de Chi-Cuadrado, Exacto de Fisher y t-Student. Posteriormente, se realizó una regresión logística que permitió examinar las variables de mayor influencia en relación con las pérdidas sensoriales aisladas y DPS (Odds ratio). Las pérdidas sensoriales resultaron ser importantes y comprometen la capacidad funcional, afectan el desempeño de las actividades básicas y restringen la movilidad. La viudez asociada con la doble pérdida fue un hallazgo sin precedentes. Para este grupo, la edad avanzada fue un factor predictivo importante para la aparición de pérdidas sensoriales, especialmente la Doble Pérdida Sensorial y tener una pérdida auditiva o visual previa aumenta el riesgo para el desarrollo de esta.

Palabras clave: Asistencia a los ancianos; Pérdida auditiva; Ceguera; Doble pérdida sensorial; Personas con discapacidad.

\section{Introdução}

O mundo vem enfrentando vários desafios na área da saúde, tanto relacionados ao rápido e intenso envelhecimento da população, quanto ao incremento na prevalência de doenças crônicas não transmissíveis (DCNT). Estas são um grupo de enfermidades de longa evolução, acompanhadas por alterações degenerativas em vários órgãos e sistemas do corpo humano que levam a lesões irreversíveis e complicações que determinam variáveis graus de incapacidade e deficiências (Organização Mundial de Saúde [OMS], 2015).

Frente a estes desafios, no Brasil, o documento "Idosos na Saúde Suplementar: uma urgência para a saúde da sociedade e sustentabilidade do setor" da Agência Nacional de Saúde, ANS, ressalta a premência do desenvolvimento e implantação pelas Operadoras de Saúde -OPS- de mecanismo de identificação de riscos e estratificações das condições de saúde no grupo populacional idoso. Assim, além da existência de planos de cuidado individualizados, pensados de acordo com a presença ou não de DCNT e de síndromes especificas da população geriátrica, o setor atenderia às necessidades diferenciadas deste grupo populacional (Manso, 2017).

Por síndromes geriátricas, entende-se um conjunto de sinais e sintomas que decorrem das DCNT com elevada prevalência em idosos, comprometendo a capacidade funcional- independência e autonomia- deste segmento populacional. A somatória de agravos causados por doenças, aliados às modificações fisiológicas comuns ao envelhecer humano, encontram-se na gênese destas síndromes, porém, estas podem ser potencializadas/ desencadeadas por questões sociais, psíquicas e, inclusive, iatrogênicas. Dentre as síndromes geriátricas destaca-se a incapacidade comunicativa relacionada tanto à perda visual quanto auditiva (Organização Panamericana de Saúde [OPAS], 2012).

Comunicação é a capacidade de estabelecer relacionamento com o meio e, depende da visão, audição e fala. A perda dessas funções pode ser causa de outras síndromes geriátricas tais como: incapacidade cognitiva, instabilidade postural e imobilidade (OPAS, 2012).

Entretanto, até agora, pouca atenção foi dada à avaliação de pessoas idosas com incapacidade comunicativa, com destaque para aquelas portadoras de dupla perda sensorial, principalmente no setor privado de saúde brasileiro, e que representam uma prioridade de pesquisa e intervenções, tanto curativas quanto preventivas. A dupla perda sensorial (DPS) refere-se à presença de perda auditiva e visual concomitantemente e pode variar de acordo com seu tempo de início, severidade e ordem de ocorrência (Wittich \& Gagné, 2016).

No contexto da literatura internacional, encontram-se estudos sobre como esta dupla perda diminui a comunicação e o bem estar entre as pessoas idosas, causando isolamento social, depressão, dependência e comprometimento cognitivo, aliados à diminuição da capacidade em realizar atividades da vida diária, declínio funcional e decréscimo da mobilidade (Guthrie, Declercq, Finne-Soveri, Fries, Hirdes, 2016). Além disso, também são relatados piora da autopercepção de saúde (Ribeiro, 
Matozinhos, Guimarães, Couto, Azevedo, 2018) e aumento do risco de mortalidade e quedas (Gopinath, McMahon, Burlutzky, \& Mitchell, 2016)

No entanto, na literatura brasileira existem poucos estudos que avaliam as necessidades específicas das pessoas idosas com DPS, notando-se uma lacuna. A colaboração multidisciplinar é essencial para compreender os fatores morfológicos, químicos, psicológicos, perceptuais e cognitivos envolvidos na DPS e minimizar suas consequências, garantindo melhores cuidados e qualidade de vida da pessoa idosa.

Tendo por base o exposto, propôs-se o presente estudo, que teve como objetivos, ao comparar grupos de idosos sem e com perdas sensoriais (visual, auditiva e DPS), primeiro, verificar a interação das perdas sensoriais com variáveis relacionadas à capacidade funcional e, segundo, detectar quais fatores influenciam a presença de DPS em um grupo de pessoas idosas vinculadas ao setor de saúde privado brasileiro.

\section{Metodologia}

Como destacam Pereira et al. (2018), esta é uma pesquisa quantitativa, transversal, exploratória, realizada entre o segundo semestre de 2018 e primeiro de 2019 com 314 idosos não institucionalizados e vinculados a um programa de gerenciamento de doenças crônicas (GDC) patrocinado por uma operadora de planos de saúde localizada na cidade de São Paulo, local de moradia destas pessoas.

Como mencionado, o setor de saúde suplementar brasileiro vem sendo estimulado, pela agência reguladora do setor, a implantar programas que permitam melhorar a qualidade de vida de seus usuários e atuar com promoção da saúde e prevenção de riscos e doenças, principalmente voltados para a população idosa (Manso, 2017).

A agência ANS destaca que a maioria das operadoras de planos de saúde executam estes programas por meio da metodologia desenvolvida nos E.U.A. e conhecida como gerenciamento de doenças crônicas (GDC). O GDC busca estimular o autocuidado mediante o aumento da responsabilização do enfermo por seu tratamento (Manso, Osti, Borrozino, \& Maresti, 2019)

No caso desta pesquisa, os idosos que participam do programa de GDC patrocinado por uma operadora de saúde, recebem regularmente visitas de enfermeiras que acompanham seu estado de saúde e trabalham a prevenção de riscos para doenças crônicas, quais sejam estímulo à atividade física e à alimentação saudável, controle da pressão arterial e peso, incentivo à cessação do hábito de fumar, entre outros. Estas visitas têm periodicidade mensal, porém, dependendo da complexidade do caso, podem sofrer modificações na frequência e incluir outros profissionais tais como fonoaudiólogos como exemplos.

As pessoas idosas participantes aderem espontaneamente ao programa, desde que tenham um diagnóstico confirmado de DCNT e estejam em tratamento médico. Não há limite temporal para permanência no programa, sendo que os médicos que assistem a estas pessoas não tem relação com a equipe de GDC e são de livre escolha pelos usuários.

O cálculo amostral para esta pesquisa foi realizado para o total de 1726 idosos participantes do programa de GDC, através do Teorema do Limite Central, considerando-se intervalo de confiança de 95\% e erro amostral de 5\%. Assim, foram sorteadas aleatoriamente 314 pessoas idosas dentre os assistidos. Foram excluídas pessoas acamadas e/ou com déficits neurológicos e cognitivos que não permitissem a realização dos testes propostos pelos pesquisadores.

A pesquisa foi conduzida no domicílio destas pessoas idosas, após prévio agendamento telefônico. Na visita, o objetivo da pesquisa foi explicado aos idosos, ressaltando-se a voluntariedade de participação e que, caso houvesse recusa, que esta não afetaria sua participação no programa ou no atendimento do plano de saúde. Após assinatura do Termo de Consentimento, foram aplicados:

a- Questionário sociodemográfico para coleta das variáveis idade, sexo, estado civil; 
b- Inquérito sobre para quais DCNT faziam tratamento e número de medicamentos de uso contínuo;

c- Pergunta sobre a presença de perda visual e/ou auditiva diagnosticada e em acompanhamento por médico ou fonoaudiólogo;

d- Aplicação da escala de Katz e Lawton (Dias \& Rodrigues, 2015) para avaliação das Atividades Básicas de Vida Diária (ABVD) e Instrumentais de Vida Diária (AIVD), nesta ordem;

e- Realização do Teste de Timed Up and Go (TUG) para verificação de risco de quedas (Leis \& Manso, 2015)

f- Escala de Depressão Geriátrica 30 itens (EDG) de Yesavage para sintomas depressivos e Miniexame do Estado Mental (MEEM) para triagem cognitiva (Gomes \& Martins, 2015).

As informações obtidas foram registradas em um banco de dados próprio do estudo. A análise estatística foi conduzida através do software Statistical Package for Social Sciences (SPSS®). As análises descritivas apresentam frequências absolutas (n) e frequências relativas (\%) para as variáveis qualitativas; para as quantitativas, se empregam média, mediana, desvio padrão, além dos valores mínimo e máximo. Este estudo permitiu a descrição do grupo como um todo.

A seguir, o grupo de idosos foi segmentado a fim de atingir os objetivos da pesquisa. Foram realizadas comparações entre os grupos: (i) com e sem perda da acuidade auditiva, (ii) com e sem perda da acuidade visual e (iii) com e sem DPS.

Para estas confrontações, após análise da normalidade pelo teste de Shapiro-Wilk $(p<0,05)$, recorreu-se, para as variáveis qualitativas, ao teste Qui-Quadrado ou Exato de Fisher, a depender do número de observações. Já as variáveis quantitativas foram comparadas através dos testes t-Student (duas categorias). Regressão logística foi então realizada mediante o método Stepwise foward, que não inclui no modelo variáveis que, analisadas conjuntamente, apresentavam-se sem significância estatística $(\mathrm{p}>0,05)$. Esta última possibilitou examinar as variáveis com maior influência em relação às perdas sensoriais isoladas e à DPS (Odds ratio). O nível de significância adotado para todos os testes foi de 5\%.

Esta pesquisa recebeu aprovação para sua realização pelo Comitê de Ética em Pesquisa, sob o parecer n 2.626.054.

\section{Resultados}

Participaram da pesquisa 314 pessoas idosas, com idade média de 78,5 anos ( $\mathrm{S}=7,3)$, mediana 77 anos, idade mínima de 67 e máxima de 104 anos. Destas, 212 (67,5\%) são mulheres e 102 (32,5\%) homens. São casados(as) 156 destes idosos (49,7\%), seguidos pelos viúvos(as) ( $\mathrm{n}=127,40,4 \%)$. Demais estados civis foram citados por 31 pessoas $(9,9 \%)$.

Hipertensão Arterial Sistêmica ( $\mathrm{n}=224,71,3 \%)$ foi a doença mais prevalente neste grupo, seguida por Artrose ( $\mathrm{n}=114$, 36,3\%), Doenças Pulmonares Obstrutivas Crônicas ( $\mathrm{n}=84,26,7 \%)$, Doenças Coronarianas $(\mathrm{n}=80,25,4 \%)$, Cânceres $(\mathrm{n}=67$, 18,6\%), Diabetes Mellitus ( $\mathrm{n}=66,21,0 \%)$, Acidente Vasculoencefálico $(\mathrm{n}=42,11,6 \%)$, dentre outras. O número médio de doenças encontrado foi de 6,1 doença/idoso ( $s=2,7$; mediana 6 ), variando entre uma e 16 doenças diagnosticadas. Fazem uso de mais de 5 medicamentos de uso contínuo por dia 68 (21,7\%) destes idosos.

Quanto ao desempenho nas ABVD, 299 (95,2\%) destas pessoas são independentes, mas, quando analisadas as AIVD, este número diminui para 238 (75,8\%). Dos idosos pesquisados, $51(16,2 \%)$ apresentaram pontos de corte ao MEEM indicativos de déficits cognitivos. Pontuaram com sintomas depressivos na EDG 19 idosos (6,1\%). Realizado o TUG, 199 $(63,4 \%)$ não apresentaram dificuldade de mobilidade.

A perda de acuidade auditiva diagnosticada foi encontrada em 129 destes idosos (41\%); perda visual em 199 (63,3\%) e DPS (visual e auditiva) em 99 (31,5\%).

As Tabelas 1, 2 e 3 apresentam as variáveis qualitativas que se associaram com significância estatística para estes grupos. As variáveis não exibidas nas tabelas não mostraram associação significante com ter algum tipo de perda sensorial (sexo; tipo e número de doenças; uso e número de medicamentos utilizados; presença de sintomas depressivos avaliados pela EDG). 
Observa-se, na Tabela 1, que ter perda auditiva diagnosticada associou-se com a presença de risco cognitivo (probabilidade de demência), maior dependência para realização de AIVD, maior dificuldade na mobilidade e com a presença de perda de acuidade visual.

Tabela 1: Variáveis e valores de p para idosos com e sem perda auditiva, grupo de pessoas idosas vinculadas a um programa de GDC, São Paulo, 2018-2019.

\begin{tabular}{|c|c|c|c|c|c|c|}
\hline \multirow{3}{*}{ Variável } & & \multicolumn{4}{|c|}{ ACUIDADE AUDITIVA } & \multirow{3}{*}{ p-valor } \\
\hline & & \multicolumn{2}{|c|}{ Sem perda } & \multicolumn{2}{|c|}{ Com perda } & \\
\hline & & $\mathrm{n}$ & $\%$ & $\mathrm{n}$ & $\%$ & \\
\hline \multirow{4}{*}{$A I V D$} & Dependência Leve & 5 & $2,7 \%$ & 13 & $10,1 \%$ & \multirow{4}{*}{$<0,001$} \\
\hline & Dependência Moderada & 17 & $9,2 \%$ & 24 & $18,6 \%$ & \\
\hline & Dependência Grave & 7 & $3,8 \%$ & 10 & $7,8 \%$ & \\
\hline & Independente & 156 & $84,3 \%$ & 82 & $63,6 \%$ & \\
\hline \multirow{2}{*}{ MEEM } & Normal & 162 & $87,6 \%$ & 101 & $78,3 \%$ & \multirow{2}{*}{0,028} \\
\hline & Risco para demência & 23 & $12,4 \%$ & 28 & $21,7 \%$ & \\
\hline \multirow{4}{*}{ RISCO DE QUEDA } & Sem dificuldade de mobilidade & 128 & $69,2 \%$ & 71 & $55,0 \%$ & \multirow{4}{*}{0,022} \\
\hline & Leve dificuldade de mobilidade & 43 & $23,2 \%$ & 35 & $27,1 \%$ & \\
\hline & $\begin{array}{l}\text { Dificuldade de mobilidade } \\
\text { moderada }\end{array}$ & 7 & $3,8 \%$ & 11 & $8,5 \%$ & \\
\hline & Dificuldade de mobilidade grave & 7 & $3,8 \%$ & 12 & $9,3 \%$ & \\
\hline \multirow{2}{*}{ ACUIDADE VISUAL } & Sem perda & 85 & $45,9 \%$ & 30 & $23,3 \%$ & \multirow{2}{*}{$<0,001$} \\
\hline & Com perda & 100 & $54,1 \%$ & 99 & $76,7 \%$ & \\
\hline
\end{tabular}

Fonte: Autores.

Já na Tabela 2, se evidencia que ter perda visual associou-se à viuvez, à dependência nas AIVD, à dificuldade de mobilidade e à perda auditiva. 
Tabela 2: Variáveis e valores de p para idosos com e sem perda visual, grupo de pessoas idosas vinculadas a um programa de GDC, São Paulo, 2018-2019.

\begin{tabular}{|c|c|c|c|c|c|c|}
\hline \multirow[b]{3}{*}{ Variável } & & \multicolumn{4}{|c|}{ ACUIDADE VISUAL } & \multirow{3}{*}{ p-valor } \\
\hline & & \multicolumn{2}{|c|}{ Sem perda } & \multicolumn{2}{|c|}{ Com perda } & \\
\hline & & $\mathrm{n}$ & $\%$ & $\mathrm{n}$ & $\%$ & \\
\hline \multirow{4}{*}{ Estado Civil } & Relação Estável & 65 & $56,5 \%$ & 91 & $45,7 \%$ & \multirow{4}{*}{0,005} \\
\hline & Separado(a) & 12 & $10,4 \%$ & 7 & $3,5 \%$ & \\
\hline & Solteiro(a) & 4 & $3,5 \%$ & 8 & $4,0 \%$ & \\
\hline & Viúvo(a) & 34 & $29,6 \%$ & 93 & $46,7 \%$ & \\
\hline \multirow{4}{*}{$A I V D$} & Dependência Leve & 1 &, $9 \%$ & 17 & $8,5 \%$ & \multirow{4}{*}{$<0,001$} \\
\hline & Dependência Moderada & 8 & $7,0 \%$ & 33 & $16,6 \%$ & \\
\hline & Dependência Grave & 1 &, $9 \%$ & 16 & $8,0 \%$ & \\
\hline & Independente & 105 & $91,3 \%$ & 133 & $66,8 \%$ & \\
\hline \multirow{4}{*}{ RISCO DE QUEDA } & Sem dificuldade de mobilidade & 92 & $80,0 \%$ & 107 & $53,8 \%$ & \multirow{4}{*}{$<0,001$} \\
\hline & Leve dificuldade de mobilidade & 16 & $13,9 \%$ & 62 & $31,2 \%$ & \\
\hline & $\begin{array}{l}\text { Dificuldade de mobilidade } \\
\text { moderada }\end{array}$ & 6 & $5,2 \%$ & 12 & $6,0 \%$ & \\
\hline & Dificuldade de mobilidade grave & 1 &, $9 \%$ & 18 & $9,0 \%$ & \\
\hline \multirow{2}{*}{ ACUIDADE AUDITIVA } & Sem perda & 85 & $73,9 \%$ & 100 & $50,3 \%$ & \multirow{2}{*}{$<0,001$} \\
\hline & Com perda & 30 & $26,1 \%$ & 99 & $49,7 \%$ & \\
\hline
\end{tabular}

Fonte: Autores.

Por sua vez, na Tabela 3 verifica-se que a DPS se relacionou com viuvez, maior dependência tanto nas ABVD quanto AIVD e dificuldade de mobilidade. 
Research, Society and Development, v. 10, n. 2, e16410212287, 2021

(CC BY 4.0) | ISSN 2525-3409 | DOI: http://dx.doi.org/10.33448/rsd-v10i2.12287

Tabela 3: Variáveis e valores de p para idosos com e sem DPS, grupo de pessoas idosas vinculadas a um programa de GDC,

São Paulo, 2018-2019.

\begin{tabular}{|c|c|c|c|c|c|c|}
\hline \multirow[b]{3}{*}{ Variável } & & \multicolumn{4}{|c|}{ DUPLA PERDA } & \multirow{3}{*}{ p-valor } \\
\hline & & \multicolumn{2}{|c|}{ Não } & \multicolumn{2}{|c|}{ Sim } & \\
\hline & & $\mathrm{n}$ & $\%$ & $\mathrm{n}$ & $\%$ & \\
\hline \multirow{4}{*}{ Estado Civil } & Relação Estável & 121 & $56,3 \%$ & 35 & $35,4 \%$ & \multirow{4}{*}{0,001} \\
\hline & Separado(a) & 15 & $7,0 \%$ & 4 & $4,0 \%$ & \\
\hline & Solteiro(a) & 7 & $3,3 \%$ & 5 & $5,1 \%$ & \\
\hline & Viúvo(a) & 72 & $33,5 \%$ & 55 & $55,6 \%$ & \\
\hline \multirow{4}{*}{$A B V D$} & Dependência Moderada & 3 & $1,4 \%$ & 6 & $6,1 \%$ & \multirow{4}{*}{0,020} \\
\hline & Dependência Severa & 3 & $1,4 \%$ & 1 & $1,0 \%$ & \\
\hline & Dependência Total & 0 & $0,0 \%$ & 2 & $2,0 \%$ & \\
\hline & Independente & 209 & $97,2 \%$ & 90 & $90,9 \%$ & \\
\hline \multirow{4}{*}{ AVDI } & Dependência Leve & 6 & $2,8 \%$ & 12 & $12,1 \%$ & \multirow{4}{*}{$<0,001$} \\
\hline & Dependência Moderada & 22 & $10,2 \%$ & 19 & $19,2 \%$ & \\
\hline & Dependência Grave & 7 & $3,3 \%$ & 10 & $10,1 \%$ & \\
\hline & Independente & 180 & $83,7 \%$ & 58 & $58,6 \%$ & \\
\hline \multirow{4}{*}{ TIME UP GO } & Sem dificuldade de mobilidade & 151 & $70,2 \%$ & 48 & $48,5 \%$ & \multirow{4}{*}{0,001} \\
\hline & Leve dificuldade de mobilidade & 46 & $21,4 \%$ & 32 & $32,3 \%$ & \\
\hline & $\begin{array}{l}\text { Dificuldade de mobilidade } \\
\text { moderada }\end{array}$ & 10 & $4,7 \%$ & 8 & $8,1 \%$ & \\
\hline & Dificuldade de mobilidade grave & 8 & $3,7 \%$ & 11 & $11,1 \%$ & \\
\hline
\end{tabular}

Fonte: Autores.

Atentou-se ainda para a interrelação entre idade, variável quantitativa, e ter alguma perda sensorial, como se observa na Tabela 4. 
Tabela 4: Idade e valores de p segundo tipo de perda sensorial, grupo de pessoas idosas vinculadas a um programa de GDC, São Paulo, 2018-2019.

\begin{tabular}{|c|c|c|c|c|c|c|c|c|}
\hline \multirow{2}{*}{$\begin{array}{l}\text { Tipo de } \\
\text { perda }\end{array}$} & & \multicolumn{6}{|c|}{ Idade } & \multirow{2}{*}{$\begin{array}{c}\mathrm{p}- \\
\text { valor }\end{array}$} \\
\hline & & $\mathrm{n}$ & média & mediana & $\mathrm{S}^{*}$ & mínimo & máximo & \\
\hline \multirow{2}{*}{$\begin{array}{l}\text { Acuidade } \\
\text { auditiva }\end{array}$} & Sem perda & 185 & 76,5 & 76 & 6,2 & 67 & 102 & $<0,001$ \\
\hline & Com perda & 129 & 81,3 & 82 & 7,8 & 67 & 104 & \\
\hline \multirow{2}{*}{$\begin{array}{l}\text { Acuidade } \\
\text { visual }\end{array}$} & Sem perda & 115 & 75,8 & 75 & 6,2 & 67 & 92 & $<0,001$ \\
\hline & Com perda & 199 & 80,1 & 79 & 7,5 & 67 & 104 & \\
\hline \multirow[t]{2}{*}{ DPS } & Sem perda & 215 & 76,8 & 76 & 6,4 & 67 & 102 & $<0,001$ \\
\hline & Com perda & 99 & 82,2 & 83 & 7,8 & 67 & 104 & \\
\hline
\end{tabular}

Fonte: Autores.

Quanto às análises multivariadas de regressão, estas mostraram que apenas idade e acuidade visual inadequada influenciam a perda auditiva, como se observa na Tabela 5, onde também são apresentados os dados relacionados à perda visual e à dupla perda sensorial. Verifica-se que idade e acuidade auditiva comprometida influenciam a perda visual e somente idade relaciona-se com dupla perda sensorial.

Desta forma, para este grupo de idosos, a cada um ano a mais vivido, há 1,10 chances de ter perda auditiva; 1,08 chances de ter perda visual e 1,11 maior probabilidade de apresentar dupla perda sensorial. Ter perda auditiva inadequada aumenta em 2,08 a chance de ter perda visual e, esta última, quando presente, eleva em 2,02 vezes a possibilidade de perda auditiva.

Tabela 5: Modelo de regressão logística (IC 95\%), idade, grupo de idosos vinculados a um programa de GDC, São Paulo, 2018-2019.

\begin{tabular}{lcccc}
\hline \multicolumn{1}{c}{ Tipo de perda } & \multicolumn{3}{c}{ Idade } & p-valor \\
\hline & OR & Limite inferior & Limite superior & \\
\hline Perda visual & 1,10 & 1,06 & 1,14 & $<0,001$ \\
Perda auditiva & 1,08 & 1,04 & 1,12 & $<0,001$ \\
DPS & 1,11 & 1,07 & 1,16 & $<0,001$ \\
\hline
\end{tabular}

Fonte: Autores.

\section{Discussão}

A distribuição sociodemográfica, quanto à idade, sexo e estado civil encontrada para este grupo de idosos aqui descrito não difere do que a literatura costuma descrever para pessoas idosas vinculadas a planos de saúde no Brasil. Apesar do envelhecimento populacional do país ser importante e veloz, sabe-se que o setor de saúde privado suplementar, que congrega 
as OPS, apresenta-se mais envelhecido que a média da população do país (Instituto de Estudos de Saúde Suplementar [IESS], 2020).

Sobre as DCNT encontradas para o grupo, não causou estranheza ser a hipertensão arterial a mais prevalente. A literatura destaca que a grande maioria dos idosos brasileiros tem hipertensão e que esta frequência aumenta conforme aumenta a idade. Já quanto ao diabetes, estudos feitos com usuários de planos de saúde no país afirmam ser esta a segunda doença mais encontrada, em associação ou não com a hipertensão arterial (Ministério da Saúde, 2018). Este fato é relevante, pois se sabe que a presença de hipertensão arterial e/ou diabetes pode aumentar o risco de alterações auditivas e visuais (Shih-Wei, Weng \& Chou, 2012).

Quanto à utilização de medicamentos, pesquisas demonstram que idosos usuários de OPS apresentam elevada utilização de medicamentos quando comparados às pessoas da mesma idade frequentadores do sistema público de saúde. Este fato é atribuído ao uso indiscriminado de especialistas que não se conversam, à inexistência de prontuário único e fragmentação do cuidado que caracteriza este setor de atenção à saúde (Agência Nacional de Saúde Suplementar [ANS], 2016).

Há poucas pesquisas no Brasil com pessoas idosas usuárias de planos de saúde privados, apesar do número destas vir aumentando paulatinamente. As existentes mostram um perfil de capacidade funcional quanto ao desempenho de ABVD e AIVD semelhantes ao aqui encontrado, o qual segue o estabelecido na literatura, onde se observa que as pessoas idosas primeiramente apresentam comprometimento no desempenho das AIVD e, posteriormente, nas ABVD, as últimas a serem comprometidas pelas síndromes geriátricas (Manso, Osti, Borrozino, \& Maresti, 2019)

Ressalta-se o encontro de 51\% destes idosos em risco de déficits cognitivos, ou seja, em risco para desenvolvimento de síndromes demenciais. A maioria das pesquisas feitas no Brasil com o MEEM, instrumento de rastreio preconizado pelo Ministério da Saúde aqui utilizado, refere-se a idosos usuários do sistema público de saúde, com nível de escolaridade baixo, o que dificulta comparações. Entretanto, quando estudadas populações com maior nível de escolaridade, a ocorrência mostra-se em valores menores ao encontrado para este grupo (Fernandes \& Andrade, 2017).

Notou-se poucas pessoas idosas com sintomas depressivos neste grupo, quando comparado ao citado na literatura, fato que deve ser mais bem estudado (Manso, Osti, Fé, Souza, Pimenta, Netto, 2021). A maioria das pessoas do grupo apresentava boa mobilidade, corroborando outras pesquisas com grupos etários semelhantes (Silva, Oliveira, Martins, Martins, Garcia, Souza, 2019).

Em relação ao número de pessoas com perdas sensoriais (auditiva, visual e DPS), este ficou próximo ao encontrado para grupo populacionais de outros países (Haanes, Kirkevold, Horgen, Hoffos, Eilertsen, 2014).

A relação encontrada entre a presença de risco para desencadeamento de demências ou risco cognitivo e perda auditiva acompanha o que é destacado pela literatura. Como comentado, perdas sensoriais, auditivas e visuais, estão associadas, quando presentes em idosos, a múltiplas morbidades, porém se destaca o deterioro cognitivo relacionado à perda auditiva. A The Lancet Comission (2017), avaliando fatores de risco para o transtorno neuro-cognitivo (síndromes demenciais), estima que a perda auditiva contribui em 8\% para o possível aparecimento ou aceleração do déficit cognitivo, mas ressalta que este pode ser evitado através da reabilitação auditiva precoce e pelo monitoramento de pessoas com mais de 60 anos, população essa que é de risco para a instalação da perda auditiva decorrente do próprio envelhecer (Pinto, Veríssimo \& Malva, 2019).

A fragilidade sensorial dos idosos deve sempre ser considerada pois, como o aqui encontrado, as perda visuais e auditivas e, principalmente, a presença de DPS, parecem ser marcadores significativos para a dependência no desempenho das atividades de vida diária. No presente estudo, os idosos com DPS se mostraram mais dependentes tantos nas ABVD quanto nas AIVD do que os idosos com déficit visual ou auditivo isolados. Este comportamento aparecia em atividades tais como: uso do 
telefone, fazer compras, responsabilizar-se pelos medicamentos, usar o transporte, cuidar da casa, usar o dinheiro e/ou preparar as refeições.

A DPS, quando presente, parece exigir destes idosos, maior integridade física e cognitiva para todas as atividades de vida diária, fato corroborado pelos achados da literatura (Gopinath, McMahon, Burlutisky, \& Mitchel, 2016). Este achado também confirma a necessidade de que os profissionais que atuam com esta população se preocupem com as perdas sensoriais neste segmento etário, cuja presença pode contribuir negativamente não só para a manutenção da integridade cognitiva desta população, mas para acelerar a dependência, fato nem sempre considerado pelos gestores de políticas de saúde, como ressalva a literatura (Pichora-Fuller, Mick, \& Reed, 2015).

Outra questão observada nesta população, diz respeito à degradação dos principais sistemas sensoriais, como propriocepção, sistema vestibular e visão, que pode ser um fator que contribui para o declínio da estabilidade da marcha em idosos. Margutti e Momensohn-Santos (2019) estudando o risco de queda em um grupo de idosos ativos, mostraram que, dos 71 participantes, 29,6\% apresentaram evidência de associação entre risco de queda e presença de DPS (valor-p = 0,001). A análise de regressão logística mostrou que os participantes com DPS apresentavam 6,13 vezes a chance para risco de queda quando comparados com os participantes que não apresentavam deficiência sensorial.

Para o grupo de idosos aqui pesquisado, a análise multivariada não evidenciou associação com o risco de quedas, mas, o estudo bivariado trouxe associação estatisticamente significativa entre dificuldade de mobilidade e as perdas sensoriais encontradas neste grupo. A redução de mobilidade é um fator causal importante para quedas em idosos, uma das síndromes geriátricas mais temidas pelas complicações advindas do evento, tais como perda de independência, isolamento social, fraturas, depressão, dentre outras (Neto et al., 2018).

Um achado inédito encontrado para este grupo de pessoas idosas foi a associação entre viuvez e a presença de DPS, fato ainda não descrito em literatura, a ser mais bem estudado. Trata-se de fator extremamente preocupante, pois estudos mostram que o idoso que mora sozinho, ou em casas onde há pouca interação comunicativa, tendem a ter sua comunicação e cognição comprometidas (Amieva \& Ouvrard, 2020; Shukla et al 2020). Pode-se afirmar que se instala um círculo vicioso, onde as perdas sensoriais potencializam o isolamento social do idoso, o qual está relacionado com incremento tantos destas perdas, quanto da probabilidade de desenvolvimento de síndromes demenciais.

As análises multivariadas de regressão, mostraram a importância do avançar da idade no aparecimento de perdas sensoriais para este grupo, fato destacado pela literatura (Haanes, Kirkevold, Horgen, Hoffos, Eilertsen, 2014). Além disto, para este grupo, apresentar uma perda sensorial isolada, seja auditiva ou visual, aumenta a probabilidade de DPS.

Sabe-se que no mundo e no Brasil, o grupo de idosos que mais tem aumentado em número é o de pessoas maiores de 80 anos, indivíduos mais propensos a experimentar DPS e seus desafios associados. De acordo com o perfil dos americanos mais velhos, realizado em 2017, nos EUA, projeta-se que a população deste país com mais de 85 anos terá um aumento de 129\%, passando de 6,4 milhões em 2016 para 14,6 milhões até 2040, e que em 2030 aproximadamente 14 milhões de adultos com mais de 70 anos desenvolverão DPS (National Center for Health Statistics, 2017).

$\mathrm{Na}$ Holanda, um estudo estimou que a DPS adquirida foi mais comum em pessoas com mais de 85 anos de idade (Vaal et al, 2007). Estudo realizado em três serviços de reabilitação em Montreal, no Canadá, alcançou resultados semelhantes e demonstrou que a maioria desse grupo de pessoas idosas possuía visão e audição residuais que poderiam ser maximizadas com o processo de reabilitação, a fim de restaurar as habilidades funcionais e a participação social perdidas (Wittich, Watanabe, \& Gagne, 2012).

Deve-se ressaltar que a incapacidade comunicativa vem sendo estudada em idosos a pouco tempo, daí existirem insuficientes estudos que avaliem as necessidades específicas das pessoas idosas com DPS (Tan et al., 2020). Daí, necessidades de várias ordens, como as relativas à comunicação, à orientação, à mobilidade, ao trabalho, a atividades 
socioculturais, ao acesso de informações sobre o que ocorre no mundo, todas essenciais para poder manter a autonomia pessoal e fazer com que esta pessoa possa continuar a sentir-se incluída no ambiente familiar e social, ainda serem pouco trabalhadas no setor saúde (Viljanen \& Tormäkangas \&Vestergaard, 2014).

Tradicionalmente, os serviços de reabilitação para perda de visão e perda auditiva são oferecidos separadamente em muitos países, incluindo o Brasil. Esses serviços são prestados de forma fragmentada por fonoaudiólogo/otorrinolaringologista assim como técnico em ortóptica/oftalmologista, respectivamente, com pouca interação entre as disciplinas. Kricos (2007) relatou que o estado atual da reabilitação fonoaudiológica para indivíduos com DPS, enfatiza o fornecimento de tecnologia assistiva apropriada para essa população, porém a autora sugere que uma consulta com um especialista da visão deva ser incluída nas melhores práticas para o tratamento fonoaudiológico de pacientes com DPS. Um especialista em visão pode realizar avaliações úteis para o fonoaudiólogo aplicar em idosos com DPS, como determinar a necessidade de contraste de cor/luminância nos controles do aparelho auditivo e propiciar materiais educativos aos pacientes; sugerir dispositivos de ampliação para a prática de audiologia; considerações de segurança para a mobilidade do paciente no consultório de fonoaudiologia e medidas de rastreamento da visão funcional.

A avaliação multidimensional do idoso, como o aqui realizado, é importante na prática para garantir a reabilitação efetiva de idosos com DPS, além de aumentar conhecimento sobre o impacto biopsicossocial desse comprometimento combinado na qualidade de vida dos idosos. Apesar de estudos buscarem atender à necessidade urgente de protocolos e intervenções baseados em evidência na reabilitação sensorial (Vreeken, Van Rens, Kramer, Knol, Van Nispen, 2015) acredita-se que mais pesquisas são necessárias no Brasil para determinar intervenções mais amplas de reabilitação auditiva e visual incluindo aconselhamento integrado, treino de mobilidade e acomodações ambientais que poderiam atrasar ou até interromper o declínio cognitivo e global do idoso mais velho.

\section{Conclusão}

Após a realização deste estudo foi possível constatar que, para este grupo de idosos, as perdas sensoriais são um fator importante que comprometem sua capacidade funcional e, portanto, sua qualidade de vida. A presença de perda auditiva associou-se ao risco cognitivo, como ressalta a literatura. Para os idosos com alguma perda, auditiva ou visual, ou com Dupla Perda Sensorial, a capacidade funcional mostrou maior comprometimento em relação aos idosos sem estas perdas, afetando o desempenho tanto para Atividades Básicas de Vida Diária quanto para Atividades Instrumentais de Vida Diária, além de restringir a mobilidade. A viuvez associada à DPS foi um achado inédito, que deve ser mais bem investigado por pesquisas futuras. Para este grupo, o avançar da idade foi um fator preditor importante para o surgimento de perdas sensoriais, principalmente a Dupla Perda Sensorial e ter uma perda prévia, auditiva ou visual, potencializa o risco para o desenvolvimento de DPS.

Acredita-se que estes achados possam ser uma contribuição importante para o gerenciamento e manejo da fragilidade sensorial em idosos mais velhos, principalmente aqueles ligados à saúde suplementar brasileira, como preconiza a ANS.

Como limitações ao estudo, reputa-se o próprio grupo estudado, o qual representa uma parcela da população idosa brasileira, com características sociodemográficas próprias e que fazem parte de um programa de GDC vinculado ao setor privado, o que não permite generalizações.

\section{Referências}

Amieva, H. \& Ouvrard, C. (2020). Does Treating Hearing Loss in Older Adults Improve Cognitive Outcomes? A Review. Journal of clinical medicine. 9(3), 805. https://doi.org/10.3390/jcm9030805

Agência Nacional de Saúde Suplementar. (2016). Idosos na Saúde Suplementar: uma urgência para a saúde da sociedade e sustentabilidade do setor. Rio de Janeiro: ANS. http://www.ans.gov.br/images/stories/Materiais_para_pesquisa/Materiais_por_assunto/ 
Dias, A. L., \& Rodrigues, T. C. (2015). Avaliação da Capacidade Funcional. In: Manso, M. E. G. \& Biffi, E. C. A. (Orgs.). Geriatria, Manual da Liga de Estudos do Processo de Envelhecimento. São Paulo, SP: Martinari.

Fernandes, J. S. G, \& Andrade, M. S. (2017). Revisão sobre a Doença de Alzheimer: diagnóstico, evolução e cuidados. Psic., Saúde \& Doenças.18(1):131140. Epub. http://dx.doi.org/10.15309/17psd180111

Gomes, L. M. C., \& Martins, L. N. S. L. (2015). Avaliação Cognitiva e do Humor. In: Manso, M. E. G., \& Biffi, E. C. A. (Orgs.). Geriatria, Manual da Liga de Estudos do Processo de Envelhecimento. Martinari.

Gopinath, B., McMahon, C. M., Burlutsky, G., \& Mitchell, P. (2016). Hearing and vision impairment and the 5 -year incidence of falls in older adults. Age and ageing, 45(3), 409-414. https://doi.org/10.1093/ageing/afw022

Guthrie, D. M., Declercq, A., Finne-Soveri, H., Fries, B. E., \& Hirdes J. P. (2016). The Health and Well-Being of Older Adults with Dual Sensory Impairment (DSI) in Four Countries. PloS one.11(5). https://doi.org/10.1371/journal.pone.0155073

Haanes, G.G., Kirkevold, M., Horgen, G., Hofoss, D., \& Eilertsen, G. (2014). Sensory impairments in community health care: a descriptive study of hearing and vision among elderly Norwegians living at home. J Multidiscip Healthc. 7:217-25.

Instituto de Estudos de Saúde Suplementar. IESS. (2020). NAB 53 Data base: novembro/2020 Nota de Acompanhamento de Beneficiários.

Kricos, P. B. (2007). Hearing Assistive Technology Considerations for Older Individuals With Dual Sensory Loss.Trends in Amplification . 11(4): 273-80.

Leis, D. F., \& Manso, M. E. G. (2015). Avaliação da Marcha, Equilíbrio e Atividade Física no idoso. In: Manso, M. E. G., \& Biffi, E. C. A. (Orgs.). Geriatria, Manual da Liga de Estudos do Processo de Envelhecimento. Martinari.

Manso, M. E. G, Osti, A. V., Borrozino, N. F., \& Maresti, L. T. P. (2019). Avaliação Multidimensional do Idoso: resultados em um grupo de indivíduos vinculados a uma operadora de planos de saúde. Revista Kairós: Gerontologia. 21(1): 191-211.

Manso, M. E. G, Osti, R. F. I., Fé, D. S. M., Souza, F. C. B., Pimenta, I. F., \& Netto, L. T. R. (2021). Relação entre níveis de atividade física, variáveis relacionadas à multidimensionalidade da saúde e presença de estresse percebido em um grupo de idosos na cidade de São Paulo. Research, Society and Development, 10 (1): e12710111612, 2021. DOI: http://dx.doi.org/10.33448/rsd-v10i1.11612

Manso, M. E. G. (2017). Envelhecimento, saúde do idoso e o setor de planos de saúde no Brasil Revista Kairos Gerontologia. 20(4): 135-151.

Margutti, P., \& Momensohn-Santos, T. M. (2019). Associação entre risco de queda e dupla perda sensorial em idosos ativos. Dissertação de Mestrado em Fonoaudiologia. Pontifícia Universidade Católica de São Paulo, PUC SP.

Ministério da Saúde (2018). Vigitel Brasil 2018: Saúde Suplementar: vigilância de fatores de risco e proteção para doenças crônicas por inquérito telefônico. Brasília: Ministério da Saúde, 2020.

National Center for Health Statistics (2017). National Health Interview Survey, Early Release of Selected Estimates Based on Data from the January-June 2017. State of the States in Developmental Disabilities. American Association on Intellectual and Developmental Disabilities.https://www.cdc.gov/nchs/data/nhis/earlyrelease/earlyrelease201712_01.pdf

Neto, J. A. C. et al. (2018). Percepção sobre queda e exposição de idosos a fatores de risco domiciliares. Ciênc. saúde coletiva, 23 (4). https://doi.org/10.1590/1413-81232018234.09252016

OMS. (2015). Organização Mundial de Saúde. Relatório Mundial de Envelhecimento e Saúde. Genebra, Suíça: OMS. http://apps.who.int/iris/bitstream/106 65/1 86463/1/ 9789240694811_eng.pdf.

OPAS. (2012). Organização Pan-Americana de Saúde. Atenção à Saúde do Idoso: Aspectos Conceituais. Brasília, DF: Organização Pan-Americana de Saúde. http://apsredes.org/pdf/Saude-do-Idoso-WEB1.pdf..

Pereira, A. S., Shitsuka, D. M., Parreira, F. J. \& Shitsuka, R. (2018) Licenciatura em computação. Metodologia da pesquisa científica.https://repositorio.ufsm.br/bitstream/handle/1/15824/Lic_Computacao_Metodologia-Pesquisa-Cientifica.pdf?sequence=1

Pichora-Fuller, M. K., Mick, P., \& Reed, M. (2015) Hearing, Cognition, and Healthy Aging: Social and Public Health Implications of the Links between AgeRelated Declines in Hearing and Cognition. Seminars in Hearing. 36(3):122-139.

Pinto, A. M., Veríssimo, M., \& Malva, J. (2019). Manual do Cuidador. Coimbra University Press.

Ribeiro, E. G., Matozinhos, F. P., Guimarães, G. L., Couto, A. M., Azevedo, R. S., \& Mendoza, I. Y. Q. (2018). Self-perceived health and clinical functional vulnerability of the elderly in Belo Horizonte/Minas Gerais. Revista Brasileira de Enfermagem, 71(suppl 2):860-7.

Shih-Wei, L. Y. S., Weng, S. F., \& Chou, C. W. (2012). Risk of Developing Sudden Sensorineural Hearing Loss in Diabetic Patients: A Population-Based Cohort Study, Otology \& Neurotology. 33 (9): 1482-88.

Shukla, A., Harper, M., Pedersen, E., Goman, A., Suen, J. J., Price, C., Applebaum, J., Hoyer, M., Lin, F. R., \& Reed, N. S. (2020). Hearing Loss, Loneliness, and Social Isolation: A Systematic Review. Otolaryngology-Head and Neck Surgery, 162(5), 622-633. https://doi.org/10.1177/0194599820910377 
Research, Society and Development, v. 10, n. 2, e16410212287, 2021

(CC BY 4.0) | ISSN 2525-3409 | DOI: http://dx.doi.org/10.33448/rsd-v10i2.12287

Silva, L. G. C., Oliveira, F. S. O., Martins, I. S., Martins, F. E. S., Garcia, T. F. M., \& Souza, A. C. P. A. (2019). Avaliação da funcionalidade e mobilidade de idosos comunitários na atenção primária à saúde. Revista Brasileira de Geriatria e Gerontologia. 22 (5): Epub https://doi.org/10.1590/198122562019022.190086

Tan, B., Man, R., Gan, A., Fenwick, E. K., Varadaraj, V., Swenor, B. K., Gupta, P., Wong, T. Y., Trevisan, C., Lorenzo-López, L., Millán-Calenti, J. C., Schwanke, C., Liljas, A., Al Snih, S., Tokuda, Y., \& Lamoureux, E. L. (2020). Is Sensory Loss an Understudied Risk Factor for Frailty? A Systematic Review and Meta-analysis. The journals of gerontology. 75(12), 2461-2470. https://doi.org/10.1093/gerona/glaa171

The Lancet Commissions. (2017). Dementia prevention and care. http://www.thelancet.com.

Vaal, J., Gussekloo, J., De Klerk, M. ,Frijters, D., et al. (2007). Acquired dual sensory impairment: In an estimated 30,000-35,000 people aged 55 years or over in the Netherlands. https://www.researchgate.net/publication/6204364_Acquired_dual_sensory_impairment_In_an_estimated_3000035000_people_aged_55_years_or_over_in_the_Netherlands

Viljanen, A., Tormäkangas, T., \& Vestergaard, S. (2014). Dual sensory loss and social participation in older Europeans. Eur J Ageing. 11 (2)155-67.

Vreeken, H. L.,Van Rens, G. H., Kramer, S. E., Knol, D. L., Van Nispen, R. M. (2015). Effects of a dual sensory loss protocol on hearing aid outcomes: a randomized controlled trial. Ear Hear. doi:10.1097/AUD.0000000000000153

Wittich, W., \& Gagné, J. (2016). Perceptual Aspects of Gerontechnology. https://www.researchgate.net/publication/307925988_Perc eptual_Aspects_of_Gerontechnology

Wittich, W., Watanabe, D. H., \& Gagne, J. (2012). Sensory and demographic characteristics of deaf blindness rehabilitation clients in Montreal, Canada. Ophthalmic Physiol Opt. 32:242-251. 